HRI-P-08-07-004

\title{
Minimal Seesaw Textures with Two Heavy Neutrinos
}

\author{
Srubabati Goswami ${ }^{1,2}$ * Atsushi Watanabe佣 \\ ${ }^{1}$ Harish-Chandra Research Institute, Chhatnag Road, Jhunsi, Allahabad 211 019, India, \\ ${ }^{2}$ Physical Research Laboratory, Navrangpura, Ahmedabad -380009, India,
}

\begin{abstract}
We systematically analyze the Dirac and the Majorana mass matrices in seesaw models with two heavy right-handed neutrinos. We perform thorough classification of the vanishing matrix elements which are compatible with the results from the current neutrino oscillation experiments. We include the possibility of a non-diagonal Majorana mass matrix which leads to new solutions viable with data. In a basis where the Majorana mass matrix is diagonal, these solutions imply a Dirac matrix with specific relationships amongst its elements. We find that at the level of total 4 zeros together in $m_{D}$ and $M_{R}$ the mass matrices are almost consistent with the data but one mixing angle is predicted to be unsuitable. At the next level, i.e. with total 3 zeros, only seven patterns of mass matrices describe the experimental data well. The seven solutions have testable predictions for the future neutrino experiments. In particular, each solution has definite predictions about the observation of the 1-3 leptonic mixing angle and the effective mass measured in neutrino-less double beta decay. The solutions of the mass matrices contain novel texture forms and provide new insights into the leptongeneration structure. We also discuss possible connections between these textures and the tri-bimaximal mixing to search for symmetry principles behind the mass matrix structure.
\end{abstract}

*sruba@prl.res.in

†watanabe@mri.ernet.in 


\section{Introduction}

Considerable progress in our understanding of neutrino properties have been made in the last decade. Spectacular results from neutrino oscillation experiments have established beyond doubt that neutrinos have mass and they mix [1]. For three neutrino generations the neutrino mass matrix at low energy is characterized by 9 parameters - the three masses, the three mixing angles and the three phases. The mixing matrix, usually known as the Pontecorvo-Maki-Nakagawa-Sakata (PMNS) matrix, is expressed in the standard parametrization as

$$
V=\left(\begin{array}{ccc}
c_{12} c_{13} & s_{12} c_{13} & s_{13} e^{-i \delta} \\
-s_{12} c_{23}-c_{12} s_{23} s_{13} e^{i \delta} & c_{12} c_{23}-s_{12} s_{23} s_{13} e^{i \delta} & s_{23} c_{13} \\
s_{12} s_{23}-c_{12} c_{23} s_{13} e^{i \delta} & -c_{12} s_{23}-s_{12} c_{23} s_{13} e^{i \delta} & c_{23} c_{13}
\end{array}\right),
$$

where $c_{i j}$ and $s_{i j}$ stand for $\cos \theta_{i j}$ and $\sin \theta_{i j}$. This matrix is to be multiplied from right by a diagonal phase matrix $P=\operatorname{diag}\left(1, e^{-i \rho / 2}, e^{-i \sigma / 2}\right)$ where $\rho$ and $\sigma$ denote the Majorana phases, which disappears if the neutrinos are Dirac particles. Oscillation experiments, so far have determined the two mass squared differences and two mixing angles and have provided an upper bound on the third mixing angle. The current data specify the $3 \sigma$ values of the oscillation parameters as presented in Table 1 [2].

Thus the data show that there are two independent mass scales with $\Delta m_{21}^{2} /\left|\Delta m_{31}^{2}\right|=$ 0.032 at the best-fit. Unlike quark sector where all mixing angles are small in the neutrino sector there are two large mixing angles while the third one can be small. The solar neutrino data have established that $\Delta m_{21}^{2}>0$. But the sign of the atmospheric mass scale $\Delta m_{31}^{2}$ is not yet known. According to the sign of $\Delta m_{31}^{2}$ the neutrino spectrum can have two hierarchies, normal hierarchy: $m_{3}^{2} \simeq \Delta m_{31}^{2} \gg m_{2}^{2} \simeq \Delta m_{21}^{2} \gg m_{1}^{2}$ with $\Delta m_{31}^{2}>0$ or inverted hierarchy: $m_{2}^{2} \simeq m_{1}^{2} \simeq\left|\Delta m_{31}^{2}\right| \gg m_{3}^{2}$ with $\Delta m_{31}^{2}<0$. The three neutrinos can also be quasi-degenerate with $m_{3}^{2} \simeq m_{2}^{2} \simeq m_{1}^{2} \gg \Delta m_{31}^{2}$ in which case there is no hierarchy. But one can still ask what the sign of $\Delta m_{31}^{2}$ is. Inverted hierarchy and quasi-degeneracy are very unlike to what is found in the quark sector. Even for normal hierarchy the mass ratio is much weaker than that in quark sector.

While, oscillation experiments can determine the mass squared differences and the mixing angles, information on absolute neutrino masses can come from tritium beta decay or neutrino-less double beta decay. The former gives the most direct bound on absolute neutrino masses from kinematics and the present bound is $m_{\beta}<2.3 \mathrm{eV}$ (95\% C.L.) coming from the Mainz tritium beta decay experiment [3]. In the standard parametrization (1.1), $m_{\beta}$ can be expressed as

$$
m_{\beta}=\left(c_{12}^{2} c_{13}^{2} m_{1}^{2}+s_{12}^{2} c_{13}^{2} m_{2}^{2}+s_{13}^{2} m_{3}^{2}\right)^{1 / 2} .
$$

Neutrino-less double beta decay violates Lepton number by two units and can occur if neutrinos are Majorana particles [4]. The best current limit on the effective mass, which is the absolute value of the ee element of the mass matrix, $m_{e e}$ :

$$
m_{e e}=c_{13}^{2} c_{12}^{2} m_{1}+e^{i \rho} c_{13}^{2} s_{12}^{2} m_{2}+e^{i(\sigma+2 \delta)} s_{13}^{2} m_{3}
$$

is given by measurements of ${ }^{76} \mathrm{Ge}$ by the Heidelberg-Moscow and IGEX collaboration [5, 6]

$$
\left|m_{e e}\right| \leq 0.35 \zeta \mathrm{eV},
$$




\begin{tabular}{c|c|c}
\hline \hline & best fit & $3 \sigma$ range \\
\hline$\Delta m_{21}^{2}\left[10^{-5} \mathrm{eV}^{2}\right]$ & 7.6 & $7.1-8.3$ \\
$\left|\Delta m_{31}^{2}\right|\left[10^{-3} \mathrm{eV}^{2}\right]$ & 2.4 & $2.0-2.8$ \\
\hline $\sin ^{2} \theta_{12}$ & 0.32 & $0.26-0.40$ \\
$\sin ^{2} \theta_{23}$ & 0.50 & $0.34-0.67$ \\
$\sin ^{2} \theta_{13}$ & 0.007 & $\leq 0.05$ \\
\hline \hline
\end{tabular}

Table 1: The present best-fit values and the $3 \sigma$ ranges of oscillation parameters from [2].

where $\zeta=\mathcal{O}(1)$ denotes the uncertainty coming from the nuclear physics involved in calculating the decay width of $0 \nu \beta \beta$.

Non-zero neutrino masses and mixing imply physics beyond the standard model. The most popular mechanism for giving small neutrino masses is the seesaw mechanism in which one adds heavy right-handed singlets (Type-I) [7, 8], scalar triplets (Type-II) [9] or fermion triplets (type-III) [10] to generate small neutrino masses at low scale. In the context of the type-I seesaw mechanism the light neutrino mass matrix is given as $\mathcal{M}=-m_{D} M_{R}^{-1} m_{D}^{\mathrm{T}}$, where $m_{D}$ is the Dirac mass matrix and $M_{R}$ is the Majorana mass matrix of the heavy right-handed neutrinos. Apart from the effective neutrino mass matrix $\mathcal{M}$, the low energy Lagrangian of the lepton sector also contains the charged-lepton mass matrix $M_{l}$. The lepton flavor mixing matrix is defined by the two unitary matrices which diagonalize each mass matrix: $V \equiv V_{l}^{\dagger} V_{\nu}$. If one assumes the charged-lepton mass matrix is diagonal then $V_{l}=1$.

One way to understand the form of neutrino masses is through texture zeros in the Majorana mass matrix at the low scale [11, 12. By texture zero we mean those entries which are vanishingly small as compared to some other elements. This approach had been adopted in quark sector [13] and therefore it seems plausible that this may work for the lepton sector also. The origin of such zero entries could be traced to symmetry or dynamics lying behind the Yukawa sector of the standard model, for example, the $U(1)$ symmetry by Froggatt and Neilsen [14 with supersymmetry, or other flavor symmetries [15] which include either discrete or continuous groups. The stability of texture zeros in $\mathcal{M}$ against renormalization group effects have been studied in [16].

Within the framework of the seesaw mechanism it is often considered more natural to study texture zeros appearing in the Yukawa coupling matrix $m_{D}$ and/or the righthanded Majorana mass matrix $M_{R}$ [17]. In general the seesaw framework contains more parameters compared to what can be obtained from measurements at low energy and it is not possible to fix the high energy parameters entirely from low energy data. Texture zeros in the matrices $m_{D}$ and/or $M_{R}$ can help in reducing the number of parameters, and thus strengthen the predictive power of the model.

Another way to increase the predictability and reduce the number of high scale parameters of the seesaw model is to reduce the number of right-handed neutrinos. The minimal number of right-handed neutrinos with which low energy phenomenology compatible with current data can be obtained is two [18. With one heavy neutrino after seesaw diagonalization the mass matrix at low scale is rank 1, that is, it contains two zero eigenvalues and hence is not consistent with the current data. 
In this paper we couple the two ideas and do an extensive and systematic analysis of all possible texture zeros in $m_{D}$ and $M_{R}$ in the framework of the minimal seesaw model containing two heavy right-handed neutrinos. There already exists exhaustive analysis of possible texture zeros in $m_{D}$ in the literature in the context of the minimal seesaw model [19. But most analyses considered a diagonal form for the Majorana mass matrix $M_{R}$. The possibility of a non-diagonal $M_{R}$ and related constraints on seesaw parameters have been discussed in few papers but this discussion is not exhaustive. In addition, it is not apparent that any mechanism leading to zeros in $m_{D}$ will necessarily require a diagonal form of $M_{R}$. It is thus worthwhile to take into account the full generality of $M_{R}$ and perform an exhaustive classification of the textures according to the total number of zeros of $m_{D}$ and $M_{R}$ together. By this systematic analysis, we encounter not only the textures which have been discussed in the literatures [17, 18, 19] but also new viable textures arising out of the general treatment for $M_{R}$, which have not been discussed.

It is important to keep in mind that, in a texture analysis, one should not fix the basis of the generation space in advance While it is true that one can always move from one basis to another by unitary transformations acting on the fields, without changing any physical consequences, (for example, from some general basis to the one in which $M_{R}$ is diagonal by redefining the right-handed neutrinos) this does not mean that one can always find full possibilities of textures by examination of the texture zeros in the $M_{R}$ diagonal basis. The unitary transformation for fields is just a change of the coordinate which describes a physical system, whereas the different (or independent) textures correspond to different systems, namely, different Lagrangians. A texture zero in some basis can appear as definite relations among matrix elements in other basis. For example, some texture zeros of $m_{D}$ and $M_{R}$ in $M_{R}$ non-diagonal basis is mapped to the specific relations among mass matrix elements of $m_{D}$ in $M_{R}$ diagonal basis. Thus the texture zeros are "hidden" in $M_{R}$ diagonal basis, in the sense that one cannot reach the texture forms of the general basis as long as one merely impose vanishing elements in $m_{D}$.

We classify the textures by clarifying whether each combination of textures can fit the experimental results or not. This is the only principle we will take. In particular, we do not stand on any aesthetic discussion and as long as a certain texture can fit the data, we regard it as viable even if it needs fine tuning of parameters to reproduce the observable. Since a priori, we do not know what kind of symmetry is lying behind some fine tuning, we believe this thorough analysis might bring useful results towards deeper understanding for the generation structure.

Throughout the analysis we assume that the charged-lepton mass matrix is diagonal. Because of the observed mass hierarchy of the charged-lepton masses, it is likely that the contribution to the PMNS matrix is small from the point of view of grand unification, though there are interesting possibilities of highly asymmetric forms of the charged-lepton mass matrix [20]. In this paper we do not consider these possibilities and simply assume that the neutrino sector is responsible solely for the structure of the lepton generation mixing.

The plan of the paper goes as follows. In the next section we discuss the rudimentary features of the seesaw model with two right-handed neutrinos. The following section discusses the number of possible texture zeros in the Dirac matrix $m_{D}$ and the Majorana mass matrix $M_{R}$ and then does a detailed classification of the patterns based on the number of texture zeros in $m_{D}$ and $M_{R}$ together. For each pattern we discuss the compatibility with 
the current oscillation data, prediction for the 1-3 leptonic mixing angle and the effective mass parameter constrained by neutrino-less double beta decay. We end in Section 4 by summarizing our results.

\section{The model with two right-handed neutrinos}

The leptonic part of the Yukawa interactions in presence of three left-handed and two right-handed neutrinos can be written as

$$
-\mathcal{L}=\left(Y_{\nu}\right)_{i j} \overline{N_{R_{j}}} \tilde{\phi}^{\dagger} l_{L_{i}}+\left(Y_{l}\right)_{i j} \overline{E_{R_{j}}} \phi^{\dagger} l_{L_{i}}+\frac{1}{2} \overline{N_{R_{i}}{ }^{c}}\left(M_{R}\right)_{i j} N_{R_{j}}+\text { h.c }
$$

where $\phi$ denotes $S U(2)$ higgs doublet with $\tilde{\phi}=i \sigma^{2} \phi^{*}$, the lepton doublet of flavor $i$ is denoted by $l_{L_{i}}, E_{R_{i}}$ are the right-handed charged-lepton singlets and $N_{R_{i}}$ denote the righthanded neutrino fields which are singlets under the standard model gauge group. The Yukawa coupling constants $Y_{\nu}$ and $Y_{l}$ are complex-valued $3 \times 3$ matrices. After the electroweak symmetry breaking one gets the charged-lepton mass matrix $M_{l}=v Y_{l}$ and the Dirac mass matrix for the neutrino as $m_{D}=v Y_{\nu}$ where $v$ is the vacuum expectation value of the neutral component of the higgs doublet $\phi$. The Majorana mass matrix $M_{R}$ is $2 \times 2$ complex symmetric matrix. The mass matrix for the neutral fermions can be written as

$$
M_{\nu}=\left(\begin{array}{cc}
0 & m_{D} \\
m_{D}^{\mathrm{T}} & M_{R}
\end{array}\right)
$$

The light neutrino mass matrix after the seesaw diagonalization assuming $M_{R} \gg m_{D}$ is given by

$$
\mathcal{M}=-m_{D} M_{R}^{-1} m_{D}^{\mathrm{T}}
$$

Since $M_{R}$ can be high the mass eigenvalues are naturally suppressed. In the $3+2$ model $m_{D}$ is a $3 \times 2$ matrix and $M_{R}$ is a $2 \times 2$ matrix while the light neutrino mass matrix $\mathcal{M}$ is $3 \times 3$. We note that the $3+2$ model can be considered as the limiting case of a $3+3$ model where the heaviest neutrino is extremely heavy compared to the others so that the contribution of the heaviest neutrino is negligibly small. In general for the $3 \times 2$ case there will be 9 free parameters characterizing the Yukawa matrix $m_{D}$ and 4 parameters for $M_{R}$ giving a total of 13 free parameters. Thus there is already a reduction from 24 to 13 as compared to the $3+3$ model. As we will see later for the cases of texture zeros in $m_{D}$ and $M_{R}$ the number of free parameters can be reduced even further.

In general the Majorana mass matrix $M_{R}$ is non-diagonal in the basis where the charged current is flavor diagonal. It is thus written as

$$
U_{R}^{\dagger} M_{R} U_{R}^{*}=\operatorname{diag}\left(M_{1}, M_{2}\right)
$$

One can make a basis rotation so that the right-handed Majorana mass matrix $M_{R}$ becomes diagonal by the unitary matrix $U_{R}$. However in that case the Dirac mass matrix $m_{D}$ also gets modified to $m_{D} U_{R}^{*}$. Let us denote the modified Dirac mass matrix as

$$
m_{D} U_{R}^{*} \equiv\left(\mathbf{a}_{1}, \mathbf{a}_{2}\right),
$$


where $\mathbf{a}_{1}$ and $\mathbf{a}_{2}$ are column vectors which have three elements. With this notation we can write the seesaw formula (2.3) as

$$
\mathcal{M}=-\sum_{i=1}^{2} \mathbf{a}_{i} \cdot \mathbf{a}_{i}^{\mathrm{T}} \frac{1}{M_{i}} .
$$

Note that the summation is stopped at 2 because we assume only two right-handed neutrinos take part in the seesaw mechanism. This expression highlights the most prominent feature of the seesaw mechanism with two heavy neutrinos. That is, the rank of the induced Majorana mass matrix is at most 2, which means that we have at least one massless lefthanded neutrino. Since the renormalization group running does not affect the rank of the mass matrix, it is a scale independent feature of the two right-handed seesaw framework.

The Majorana mass matrix $\mathcal{M}$ is symmetric and can in general be diagonalized as

$$
V_{\nu}^{\mathrm{T}} \mathcal{M} V_{\nu}=D_{\nu}
$$

where $D_{\nu}$ is the diagonal (real and positive) mass eigenvalues for the left-handed neutrinos: $D_{\nu}=\operatorname{diag}\left(m_{1}, m_{2}, m_{3}\right)$, and $V_{\nu}$ is an unitary matrix which includes 3 angles and 6 phases in general. The charged-lepton mass matrix $M_{l}$ is diagonalized as

$$
V_{l}^{\mathrm{T}} M_{l} U_{l}^{*}=D_{l}
$$

where $V_{l}$ and $U_{l}$ are unitary matrices which can be removed by unitary rotations of the left and the right-handed charged-leptons. The diagonal matrix $D_{l}$ denotes the observed charged-lepton masses: $D_{l}=\operatorname{diag}\left(m_{e}, m_{\mu}, m_{\tau}\right)$. The generation mixing for leptons is described by the Pontecorvo-Maki-Nakagawa-Sakata (PMNS) matrix, which is defined by the product of the two unitary matrices;

$$
V \equiv V_{l}^{\dagger} V_{\nu}
$$

A general $3 \times 3$ unitary matrix can be parameterized by three 3 angles and 6 phases. Out of 6 phases, 3 phases can be removed by the redefinition of the left-handed neutrino fields. Thus 3 angles and 3 phases can affect observables if the neutrinos are Majorana particle. In the case where the neutrinos are Dirac particle, we can further remove 2 phases by using the right-handed neutrinos, and there is only 1 phase which is responsible for $\mathrm{CP}$ violation, just as in the quark sector.

In the following discussion, we assume that the charged-lepton mass matrix is diagonal, so that $V_{l}=I$. While this treatment will not cover whole possible solutions in the lepton sector, it turns out that this is indeed a good first step to extract physics involved in the seesaw mass matrices with two right-handed neutrinos. Against the diagonal chargedlepton mass matrix, we will perform texture analysis step by step from the most minimal (maximal number of zero) level. We discuss not only successful textures, but also the textures which are not totally compatible with the experimental data. These textures can become viable with the inclusion of non-diagonal charged lepton matrices and from an understanding of which sector is inconsistent with data it will be possible to determine the form of the non-diagonal charged-lepton textures which are needed to make the "close to viable" seesaw texture acceptable. 
Finally, we would like to note that the generation indices for the lepton doublet is thus fixed in any discussions below. In particular, it should be noted that any exchanging operation for the rows of $m_{D}$ do affect physical consequences (however small they are) so that the textures which are related to each other by such exchange should be regarded as independent textures.

\section{$3 \quad$ Texture analysis}

In this section we will first consider the texture zeros of $m_{D}$ and $M_{R}$ separately and then check the total number of texture zeros together in $m_{D}$ and $M_{R}$. Here the number of the zero means the number of the independent vanishing elements in each matrix. For example, for a symmetric matrix, "1 zero" means that a diagonal element or a pair of off-diagonal components in symmetric positions are anomalously small compared to the other elements.

\subsection{Zeros of the Dirac mass matrix $m_{D}$}

First of all, let us consider the minimality of $m_{D}$, without taking into account the form of $M_{R}$. The following cases may arise:

\section{- More than 3 zeros}

It should be useful to note that if the number of vanishing elements in $m_{D}$ is $\geq 4$, it cannot lead to viable forms of the effective Majorana matrix $\mathcal{M}$. This is because if we have 4 or more than 4 zeros in $m_{D}$, there is at least one vanishing row in the Dirac mass matrix. This means that at least one left-handed neutrino is decoupled from the right-handed states so that the neutrinos can be mixed only between the other two states which are coupled with the right-handed neutrinos. We can thus exclude more than 3 zeros in $m_{D}$.

\section{- 3 zeros}

The next possibility is to consider three zeros in $m_{D}$. We have ${ }^{6} \mathrm{C}_{3}=20$ patterns of matrices as the general possibilities of 3 zero textures in $m_{D}$. They can be classified into three categories. The first one includes the matrices which have one vanishing row. There are 12 patterns of such matrices but none of them can reproduce observation, as we saw in the discussion above. The second one includes the matrices which have one vanishing column. The 2 patterns of such matrices are also not viable because the vanishing column implies that only one right-handed neutrino takes part in the seesaw mechanism, which leads to two massless states. The third category is formed by the other 6 patterns. We cannot exclude these patterns as long as we are concerned with only Dirac mass matrix $m_{D}$. An example in this category is

$$
m_{D}=\left(\begin{array}{ll}
0 & d \\
b & 0 \\
c & 0
\end{array}\right) .
$$

The other 5 patterns are obtained by permuting the rows and columns of (3.1). In the following discussions, we will examine these 6 patterns of $m_{D}$ as general possibilities of the 3 zero $m_{D}$. 


\section{- 2 zeros}

The next to minimal number of zeros is 2 . In this case we have ${ }^{6} \mathrm{C}_{2}=15$ patterns of matrices as the general possibilities. They can be classified into three categories. The first one includes the 3 matrices which have one vanishing row. As we saw in the discussion above, we need not to examine these three. The second one includes the 6 matrices which have two zero entries in the same column, for example

$$
m_{D}=\left(\begin{array}{ll}
a & 0 \\
b & 0 \\
c & f
\end{array}\right) .
$$

The other five patterns are obtained by permuting the rows and the columns of (3.2). The third category is formed by the other 6 patterns, which have two zero elements in different columns. For example,

$$
m_{D}=\left(\begin{array}{ll}
a & 0 \\
0 & e \\
c & f
\end{array}\right) .
$$

The other five patterns are obtained by permuting the rows and the columns of (3.3) (or the permutations of the rows only). The 12 matrices which belong to the last two categories are not excluded a priori. In the following discussions, we take these 12 patterns as general possibilities of the 2 zero $m_{D}$.

\subsection{Zeros of $M_{R}$}

Since we have a $2 \times 2 M_{R}$ and we include the possibility of non-diagonal $M_{R}$ we can have the following options

- 3 zeros

This gives a $M_{R}$ with all entries as zero and so this is excluded.

- 2 zeros

In this case there are two possibilities

(i) The diagonal entries are zero

$$
M_{R}=\left(\begin{array}{cc}
0 & M_{12} \\
M_{12} & 0
\end{array}\right)
$$

(ii) One diagonal entry and the off-diagonal entries are zero

$$
M_{R}=\left(\begin{array}{cc}
0 & 0 \\
0 & M_{22}
\end{array}\right) .
$$

or 


$$
M_{R}=\left(\begin{array}{cc}
M_{11} & 0 \\
0 & 0
\end{array}\right)
$$

The last two options give vanishing determinants and give rise to a state which does not receive seesaw suppression in mass. In this paper, we do not consider such exotic spectrum though it is an interesting possibility to accommodate more than two mass differences. We therefore conclude that only the option (i) can be viable.

- 1 zero

The possible options for this case are

$$
\begin{aligned}
& M_{R}=\left(\begin{array}{cc}
0 & M_{12} \\
M_{12} & M_{22}
\end{array}\right) . \\
& M_{R}=\left(\begin{array}{cc}
M_{11} & M_{12} \\
M_{12} & 0
\end{array}\right) . \\
& M_{R}=\left(\begin{array}{cc}
M_{11} & 0 \\
0 & M_{22}
\end{array}\right) .
\end{aligned}
$$

All of these matrices have non-vanishing determinant. We thus regard these three matrices as general possibilities in the following analysis.

\subsection{Zeros of $m_{D}$ and $M_{R}$ combined - almost viable forms -}

So far we have discussed the texture zeros for the Dirac mass matrix $m_{D}$ and the Majorana mass matrix $M_{R}$ separately. In this section we search for the combinations of $m_{D}$ and $M_{R}$ which are compatible with the present oscillation data, keeping the results of Section 3.1 and 3.2 in mind. We classify the combinations of the two matrices according to total number of texture zeros to be distributed in $m_{D}$ and $M_{R}$. We will find that at the 4 zero level, several textures appear to be close to perfectly viable.

Let us consider total 6 zero elements in the mass matrices $m_{D}$ and $M_{R}$ as a starting point of texture analysis. The maximum number of texture zeros admissible in $m_{D}$ is three. This leaves us with the rest of the three zeros in $M_{R}$ which is excluded. Thus there are no viable textures in the case of the total 6 zeros.

We next consider the possibility of having total 5 zeros in $m_{D}$ and $M_{R}$. According to the discussion in Section 3.1 and 3.2. we can only have the case where $m_{D}$ has 3 zeros and $M_{R}$ has 2 zeros. Then $\mathcal{M}$ is given as,

$$
\begin{aligned}
\mathcal{M} & =\left(\begin{array}{ll}
0 & d \\
b & 0 \\
c & 0
\end{array}\right)\left(\begin{array}{cc}
0 & \frac{1}{M_{12}} \\
\frac{1}{M_{12}} & 0
\end{array}\right)\left(\begin{array}{lll}
0 & b & c \\
d & 0 & 0
\end{array}\right) \\
& =\left(\begin{array}{ccc}
0 & b d & c d \\
b d & 0 & 0 \\
c d & 0 & 0
\end{array}\right) \frac{1}{M_{12}} .
\end{aligned}
$$


It should be noted that $M_{R}$ is invariant under the exchange of the generation label. By virtue of this feature, the resultant Majorana mass matrices of the other possible combinations are obtained by permuting the rows and the columns of (3.10). It is interesting to note that the texture form (3.10) can be obtained from $L_{e}-L_{\mu}-L_{\tau}$ flavor symmetry. While naive bi-maximal structure can be produced with inverted hierarchy, it is clear that the two nonzero masses are degenerate. Thus we conclude that there is no solution in the level of the total 5 zero texture.

The next step is the total 4 zero textures. In this case, there are two possibilities;

(i) 3 zeros in $m_{D}$ and 1 zero in $M_{R}$

(ii) 2 zeros in $m_{D}$ and 2 zeros in $M_{R}$

In the following we investigate the above cases in detail one by one.

\section{(i) 3 zero $m_{D}$ and 1 zero $M_{R}$}

To study this case, it is convenient to write down an example of the seesaw formula in terms of a most general $2 \times 2 M_{R}$ as

$$
\begin{aligned}
\mathcal{M} & =\left(\begin{array}{ll}
0 & d \\
b & 0 \\
c & 0
\end{array}\right)\left(\begin{array}{ll}
A & B \\
B & C
\end{array}\right)\left(\begin{array}{lll}
0 & b & c \\
d & 0 & 0
\end{array}\right) \\
& =\left(\begin{array}{lll}
d^{2} C & b d B & c d B \\
b d B & b^{2} A & b c A \\
c d B & b c A & c^{2} A
\end{array}\right),
\end{aligned}
$$

where we introduce the parameters $A, B, C$ just to simplify the notation:

$$
\left(\begin{array}{ll}
A & B \\
B & C
\end{array}\right) \equiv\left(M_{R}\right)^{-1}=\frac{1}{M_{11} M_{22}-M_{12}^{2}}\left(\begin{array}{cc}
M_{22} & -M_{12} \\
-M_{12} & M_{11}
\end{array}\right)
$$

In the following, we examine 1 zero $M_{R}$ by taking $A, B$ or $C$ to be zero in turn. As we saw in Section 3.1, there are 6 patterns of 3 zero $m_{D}$ which we should consider. Although (3.11) presents just an example of the 6 possible combinations of 3 zero $m_{D}$ and general $M_{R}$, we can study the consequences of the other 5 patterns by careful observation of (3.11).

Keeping $A, B$, and $C$ to be non-zero, we have 12 real parameters characterizing $m_{D}$ and $M_{R}$. All the 3 phases of $m_{D}$ and 2 phases of $M_{R}$ can be removed by redefining the fields and so for this case we have 7 parameters -3 real parameters for $m_{D}$ and 4 real parameters for $M_{R}$ (one of which is a phase). With no loss of generality, we can take the basis in which the matrix element $C$ has a phase. We thus regard only $\mathcal{M}_{11}$ as complex in what follows.

The most striking feature of the matrix (3.11) is that it has an eigenvector which is associated with the zero eigenvalue; that is, $\left(0,-c / \sqrt{b^{2}+c^{2}}, b / \sqrt{b^{2}+c^{2}}\right)^{\mathrm{T}}$. Note that this vector does not depend on the phase of $\mathcal{M}_{11}$. Since the zero element in this eigenvector must be interpreted as the reactor angle, the mass ordering of the neutrinos is fixed to be the inverted hierarchy. Since this matrix yields a vanishing $\theta_{13}$ it is characterized by a class 
of the discrete $Z_{2}$ symmetry [21]. The atmospheric angle is controlled by the elements $b$ and $c$. By taking $b \simeq(=) c$, we have $\theta_{23} \simeq(=) 45^{\circ}$. This corresponds to $\mu-\tau$ exchange symmetry.

We have three options to take 1 zero texture in $M_{R}$, that is, $A=0, B=0$ or $C=0$. In these options, we find that $C=0$ is the most promising texture and the other two cases are disfavored at the first glance:

- $B=0$ : This case corresponds to a 3 zero $m_{D}$ with a diagonal $M_{R}$. In this case, there is only one mixing - in the 2-3 sector and thus cannot reproduce two large mixing angles.

- $A=0$ : There are three zeros in the Majorana mass matrix $\mathcal{M}$ which is not viable with current data [11]. We note that in this case the 1-2 mixing angle will be given as

$$
\sin \theta_{12} \simeq \frac{1}{\sqrt{2}}+\frac{1}{8 \sqrt{2}} \alpha
$$

where $\alpha$ is the small parameter $\alpha \equiv \Delta m_{21}^{2} /\left|\Delta m_{31}^{2}\right|$. Thus the 1-2 angle is greater than the maximal value of $\pi / 4$ which is ruled out by solar neutrino data [22].

Note that the logic which we have used to exclude $A=0$ and $B=0$ are also applicable to the case of the other 5 patterns of $m_{D}$ because the Majorana mass matrix $\mathcal{M}$ for the other 5 textures can be obtained by permuting rows and columns of (3.11).

By setting $C=0$, we lose the sole matrix element which is responsible for the $\mathrm{CP}$ violation. This means that there is no $\mathrm{CP}$ violating phase in the lepton Yukawa Lagrangian. As we have seen the above, there is an eigenvector $\left(0,-c / \sqrt{b^{2}+c^{2}}, b / \sqrt{b^{2}+c^{2}}\right)^{\mathrm{T}}$. This fact forces the mass spectrum to be the inverted hierarchy. Moreover, we can see that it needs $b \simeq c$ to produce the observation of the atmospheric neutrinos. The inverted mass spectrum is then realized in the region where $\mathcal{M}_{12} \simeq \mathcal{M}_{13} \gg \mathcal{M}_{22} \simeq \mathcal{M}_{23} \simeq \mathcal{M}_{33}$ is satisfied. The magnitudes of the two nonzero mass eigenvalues $m_{1}$ and $m_{2}$ are controlled by the magnitude of $\mathcal{M}_{12} \simeq \mathcal{M}_{13}$ and the mass difference between these two states are ruled by $\mathcal{M}_{22} \simeq \mathcal{M}_{23} \simeq \mathcal{M}_{33}$. This means that the 1-2 rotation angle is controlled by the ratio $d B / b A$ in such a way that $\tan 2 \theta_{12} \simeq 2 d B / b A \gg 1$. Thus the solar angle will be predicted as $\simeq 45^{\circ}$. In fact, we can write down the solar angle $\sin \theta_{12}$ in terms of the mass differences as

$$
\sin \theta_{12} \simeq \frac{1}{\sqrt{2}}-\frac{1}{8 \sqrt{2}} \alpha
$$

The prediction (3.14) is due to the absence of the 1-1 matrix element in the low energy Majorana mass matrix. Although the solar angle is predicted to be smaller than $45^{\circ}$, the deviation from maximal is too small to be compatible with the data.

As for the other possible combinations, we can see the consequences immediately from the above one example. As we saw in Section 3.1. there are 6 patterns of 3 zero $m_{D}$ which we should consider, and they are related each other by permutations of the rows and the columns. The one of them is just the texture which we took in (3.11). Thus the other 5 resultant Majorana mass matrices are obtained by exchanging the rows and the columns of (3.11), and replacing the matrix element $A$ with $C$. In the case of the 2 patterns of $m_{D}$ 
which are obtained by permuting rows of $m_{D}$ in (3.11), it should be noted that we cannot replace the first row with the other one, otherwise the zero element in the mixing matrix comes in the wrong position. This means that these 2 patterns can be safely excluded.

As for the 3 patterns which are associated with exchanging the columns of $m_{D}$ in (3.11), we can discuss in the same manner as the above by replacing $A$ with $C$. We find that the Dirac mass matrix obtained by a column exchange

$$
m_{D}=\left(\begin{array}{ll}
d & 0 \\
0 & b \\
0 & c
\end{array}\right)
$$

is the most promising one. The Majorana mass matrix $\mathcal{M}$ for this case can be related to the general case considered in (3.11) by exchange of label of the right-handed neutrinos $A \leftrightarrow C$ i.e.

$$
M_{R}^{-1}=\left(\begin{array}{cc}
C & B \\
B & A
\end{array}\right)
$$

It is clear from (3.11) that after the exchange $A \leftrightarrow C$ the promising texture in this case is obtained by putting $\mathrm{A}=0$. In summary, in the case of 3 zero $m_{D}$ and 1 zero $M_{R}$, we found that the most promising texture, which can give one zero and two large mixing angles, is the $C=0$ case in (3.11) and $A=0$ with (3.15). However, these two combinations are related each other by the label exchange of the two heavy neutrinos so that these two solutions describe exactly the same physics. Over all, we conclude $C=0$ case in (3.11) (or $A=0$ with (3.15D) is the most promising texture. The predictions are very distinctive;

- The inverted hierarchy with $m_{3}=0$.

- $\theta_{13}=0$.

- No CP at all (at high energy as well as low energy).

- $\sin \theta_{12} \simeq \frac{1}{\sqrt{2}}-\frac{1}{8 \sqrt{2}} \alpha$.

The atmospheric angle $\theta_{23}$ is well controlled, and we can reproduce the best fit value $\theta_{23}=45^{\circ}$. Thus this texture, which can be obtained since we have gone beyond the assumption of a diagonal $M_{R}$, needs some correction to the 1-2 mixing angle. Such a correction can be traced to various sources. For example, it is clear that 1-2 mixing from charged-lepton sector can ameliorate the problem immediately. As another example, it might be possible to cure the problem by the renormalization group effect from some (high) energy scale (at which the texture zeros are imposed) to the electroweak scale [23].

\section{(i) 2 zero $m_{D}$ and 2 zero $M_{R}$}

Next let us explore the case of 2 zero $m_{D}$ and 2 zero $M_{R}$. As we have discussed in Section 3.2. for two zero $M_{R}$ the only allowed possibility with non-vanishing determinant is the one with zeros at the diagonal positions. Note that in a basis where $M_{R}$ is diagonal, the elements of $m_{D}$ in this basis are made out of the linear combinations of the elements in the 
original basis. Therefore texture zeros for $m_{D}$ in the new basis imply particular relations between matrix elements of $m_{D}$ in the old basis, so that the texture analysis considering zeros of $m_{D}$ and a diagonal $M_{R}$ would not include this 2 zero $m_{D}$ and 2 zero $M_{R}$ case.

As we saw in Section [3.1, we should consider two types of 2 zero $m_{D}$. Namely, the 6 patterns represented by (3.2) with the two zero entries in the same column and the other 6 patterns represented by (3.3) with the zero entries in different columns. A representative for the 6 combinations which involve the former type of $m_{D}$ is

$$
m_{D}=\left(\begin{array}{cc}
a & d \\
b & 0 \\
c & 0
\end{array}\right), \quad M_{R}^{-1}=\left(\begin{array}{cc}
0 & B \\
B & 0
\end{array}\right)
$$

However these mass matrices induce the same form of the left-handed Majorana mass matrix as that of (3.11) with $A=0$. We thus drop this combination from the list of promising texture. Furthermore, we can also drop the other 5 combinations because their effective Majorana mass matrices can be obtained by permuting rows and columns of that of (3.17). We thus conclude that there is no viable texture in this category.

For the group represented by (3.3), we have an example

$$
m_{D}=\left(\begin{array}{cc}
a & 0 \\
0 & e \\
c & f
\end{array}\right), \quad M_{R}^{-1}=\left(\begin{array}{cc}
0 & B \\
B & 0
\end{array}\right) .
$$

After the seesaw integration, we have

$$
\mathcal{M}=\left(\begin{array}{ccc}
0 & a e & a f \\
a e & 0 & c e \\
a f & c e & 2 c f
\end{array}\right) B .
$$

There remains two texture zeros at the low energy scale. We note that by the redefinition of the fields, all the phase degrees of freedom can be moved away from the Yukawa Lagrangian of the lepton sector. Thus there is no CP violating phenomena with the texture (3.18) at high energy as well as low energy.

The texture combination (3.18) can accommodate both the normal and the inverted hierarchy. We study them individually.

Normal hierarchy In the case of the normal hierarchy, the neutrino masses $m_{1}, m_{2}$ and $m_{3}$ are fixed as $m_{1}=0, m_{2}=\sqrt{\Delta m_{21}^{2}}$ and $m_{3}=\sqrt{\Delta m_{31}^{2}}$. Since we have only 3 effective parameters after the seesaw operation, we have two weak eigen-basis invariant predictions;

$$
\begin{aligned}
\frac{\tan \theta_{13}}{\sin \theta_{12}} & =\alpha^{\frac{1}{4}}, \\
\sin \theta_{23} & \simeq \alpha^{\frac{1}{4}}-\frac{1}{2} \alpha^{-\frac{1}{4}} \sin ^{2} \theta_{13},
\end{aligned}
$$

where $\alpha \equiv \Delta m_{21}^{2} / \Delta m_{31}^{2}$. Substituting $3 \sigma$ lower bound of $\alpha$ and $\sin \theta_{12}$ into (3.20), we find

$$
\sin \theta_{13}=0.20
$$


Thus with the marginal values of $\alpha$ and $\sin \theta_{12}$ we already reach just below the current upper bound on $\sin \theta_{13}$ in (3.22). Moreover, from the relation (3.21), we can see that there is an anti-correlation between $\sin \theta_{13}$ and $\sin \theta_{23}$ in the sense that for a smaller $\sin \theta_{13}$, $\sin \theta_{23}$ will be larger. Hence a conservative upper bound of $\sin \theta_{23}$ can be obtained in the limit $\theta_{13} \rightarrow 0$. Then we find

$$
\sin \theta_{23} \lesssim \alpha^{\frac{1}{4}}=0.42
$$

where we used the best fit values of the mass differences on the right-hand side. While the solar and the reactor angles are within present $3 \sigma$ data, we need some corrections to fit the atmospheric angle.

As we saw in 3.1, the other 5 patterns of Dirac mass matrices are obtained by permuting rows of (3.3). It is useful to note that the form of $M_{R}$ does not change if we exchange its rows and columns. Thus, the Dirac textures which are related with column exchange induce the same predictions and they cannot be independent solution each other. This fact reduces the number of the mass matrices which we need to examine 5 to 2 , that is, it is enough to consider 2 patterns which is obtained by exchanging 1-3 and 2-3 rows of (3.3).

For the 1-3 exchanging texture, the PMNS matrix element $\left|V_{33}\right|$ for the case of (3.3) is identified as the reactor angle. However, we can see from (3.21) that this element is approximated as

$$
\left|V_{33}\right| \simeq 1-\frac{1}{2} \alpha^{\frac{1}{2}}
$$

Thus the proper magnitude for the reactor angle cannot realized at all. We conclude that 1-3 exchanging texture from (3.3) is excluded because of this large discrepancy in $\theta_{13}$.

On the other hand, the 2-3 exchanging texture should be regarded as the same level as the texture of (3.3). The conservative upper bound (3.23) implies that the lower bound of $\left|V_{33}\right| \geq 0.82$ from the normalization condition of the third column vector in the PMNS matrix. Since the allowed range of the atmospheric angle is almost symmetric around the maximal value $45^{\circ}$, the deviations from the best fit value are the same in both textures.

Inverted hierarchy In the case of the inverted hierarchy, the masses $m_{1}, m_{2}$ and $m_{3}$ are fixed as $m_{1}=\sqrt{\left|\Delta m_{31}^{2}\right|}, m_{2}=\sqrt{\left|\Delta m_{31}^{2}\right|+\Delta m_{21}^{2}}$ and $m_{3}=0$. There are two relations among observables;

$$
\begin{aligned}
\sin \theta_{13} & \simeq \frac{1}{4 \tan \theta_{23}} \alpha \\
\sin \theta_{12} & \simeq \frac{1}{\sqrt{2}}-\frac{1}{8 \sqrt{2}} \alpha-\frac{1}{2 \sqrt{2}} \sin \theta_{13}
\end{aligned}
$$

where $\alpha \equiv \Delta m_{21}^{2} /\left|\Delta m_{31}^{2}\right|$. From (3.25), we can see that $\sin \theta_{13}$ is predicted to be small; $\sin \theta_{13} \sim \mathcal{O}\left(10^{-3}\right)$ (with the best fit values of $\theta_{23}$ and the mass differences, we have $\sin \theta_{13}=$ $\left.8.0 \times 10^{-3}\right)$. As for the solar angle, we can see from (3.26) that it becomes near the maximal value $\theta_{12} \simeq 45^{\circ}$. Thus we see that the texture (3.18) predicts nearly bi-maximal mixing with the inverted mass ordering. The deviation from the bi-maximal form is observed to be small and it is characterized by the magnitude of the reactor angle $\sin \theta_{13} \sim \mathcal{O}\left(10^{-3}\right)$. Thus this case also needs correction to the 1-2 mixing angle to become a perfectly viable. 
According to the discussion in Section 3.1, there remains 5 textures which should be examined. However, due to the 1-2 permutation invariance of $M_{C}^{-1}$, we need not try the 3 textures which are related with (3.3) by exchanging the columns. Thus it is enough to investigate the two textures which are obtained by exchanging the 1-3 and 2-3 rows of (3.3), as in the case for the normal hierarchy. For the Dirac mass matrix $m_{D}$ which is obtained by 1-3 row exchange of (3.3), the relation (3.25) implies that the small entry of $\mathcal{O}\left(10^{-3}\right)$ is sitting in $V_{33}$. Moreover, the element $\left|V_{23}\right|$ must be near maximal to fit the atmospheric neutrino data. Thus the 1-3 element $\left|V_{13}\right|$ is also near maximal, which is by no means viable. On the other hand the 2-3 exchanging texture is apparently viable because the bi-maximal mixing does not change physics under this exchange.

\subsection{Zeros of $m_{D}$ and $M_{R}$ combined - viable mass matrices -}

In this section, we explore the case where the neutrino Yukawa sector has total 3 vanishing elements. In this level, we will find textures which are totally compatible with the data and have one definite correlation among neutrino masses and mixings.

For total 3 zero case the different possibilities are

(A) 3 zero $m_{D}$ and no zero $M_{R}$

(B) 2 zero $m_{D}$ and one zero $M_{R}$

(C) 1 zero $m_{D}$ and two zero $M_{R}$

By exhausting all texture combinations in each category above, we found 7 combinations of $m_{D}$ and $M_{R}$ which are consistent with the present $3 \sigma$ data for the three generation neutrino oscillation.

Table 2. shows 7 solutions and their predictions. Besides the 7 patterns in the table, there exist other 7 solutions which can be obtained by permuting 2-3 rows of $m_{D}$ for each texture. Although these 14 patterns are independent in the sense that they are not related each other by the field rotation, the predictions are almost the same for both 7 textures. Thus we present only 7 partners in Table 2. We would like to emphasize that we are not dropping any possibilities. The result is obtained partly by general considerations and partly by direct examination of each mass matrix combination.

In the following, we build up the whole picture by discussing each of the seven cases presented in Table 2,

\subsubsection{A1. 3 zero $m_{D}$ and 0 zero $M_{R}$}

Here we discuss the solution A1, where the Dirac mass matrix $m_{D}$ has 3 zeros while the Majorana mass matrix $M_{R}$ has no-vanishing entries;

$$
m_{D}=\left(\begin{array}{ll}
0 & d \\
b & 0 \\
c & 0
\end{array}\right), \quad M_{R}^{-1}=\left(\begin{array}{ll}
A & B \\
B & C
\end{array}\right) .
$$

For this case we have 12 real parameters characterizing $m_{D}$ and $M_{R}$. All the 3 phases of $m_{D}$ and 2 phases of $M_{R}$ can be removed by redefining the fields and so for this case we 


\begin{tabular}{|c|c|c|c|c|c|}
\hline & $\begin{array}{ll}m_{D}, & M_{R}^{-1} \\
\end{array}$ & $\overline{\mathrm{NH}}$ & $\overline{\mathrm{IH}}$ & $\overline{s i n} \theta_{13}$ & $\overline{\left|m_{e e}\right|(\mathrm{eV})}$ \\
\hline A1 & $\left(\begin{array}{ll}0 & d \\
b & 0 \\
c & 0\end{array}\right),\left(\begin{array}{ll}A & B \\
B & C\end{array}\right)$ & $x$ & 0 & $\sim 0$ & $\sim 0.02$ \\
\hline B1 & $\left(\begin{array}{ll}a & d \\
b & 0 \\
c & 0\end{array}\right),\left(\begin{array}{ll}A & 0 \\
0 & C\end{array}\right)$ & $\times$ & 0 & $\sim 0$ & $\sim 0.02$ \\
\hline B2 & $\left(\begin{array}{ll}a & 0 \\
b & e \\
0 & f\end{array}\right),\left(\begin{array}{ll}A & 0 \\
0 & C\end{array}\right)$ & $\bigcirc$ & 0 & $\begin{cases}\simeq \frac{1}{2} \sin 2 \theta_{12} \tan \theta_{23} \sqrt{\alpha}, & (\mathrm{NH}) \\
\simeq \frac{1}{4} \sin 2 \theta_{12} \tan \theta_{23} \alpha, & (\mathrm{IH})\end{cases}$ & 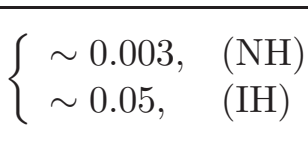 \\
\hline B3 & $\left(\begin{array}{ll}a & d \\
b & 0 \\
c & 0\end{array}\right),\left(\begin{array}{ll}A & B \\
B & 0\end{array}\right)$ & $\times$ & $\bigcirc$ & $\sim 0$ & $\sim 0.02$ \\
\hline B4 & $\left(\begin{array}{ll}a & 0 \\
0 & e \\
c & f\end{array}\right),\left(\begin{array}{ll}0 & B \\
B & C\end{array}\right)$ & $\bigcirc$ & $x$ & $\simeq \alpha^{\frac{1}{4}} \sin \theta_{12}$ & $\sim 0$ \\
\hline $\mathrm{C} 1$ & $\left(\begin{array}{ll}a & 0 \\
b & e \\
c & f\end{array}\right),\left(\begin{array}{cc}0 & B \\
B & 0\end{array}\right)$ & $\bigcirc$ & $x$ & $\simeq \alpha^{\frac{1}{4}} \sin \theta_{12}$ & $\sim 0$ \\
\hline $\mathrm{C} 2$ & $\left(\begin{array}{ll}a & d \\
b & 0 \\
c & f\end{array}\right),\left(\begin{array}{cc}0 & B \\
B & 0\end{array}\right)$ & $\times$ & $\bigcirc$ & $\simeq\left(1-\sqrt{2} \sin \theta_{12}-\frac{1}{8} \alpha\right) \cot \theta_{23}$ & $\sim 0.01$ \\
\hline
\end{tabular}

Table 2: The 7 solutions for the total 3 zero textures. The column "NH" and "IH" means the normal and the inverted hierarchy respectively. In these columns, the symbol " $\bigcirc$ " means each texture can accommodate each mass ordering, and " $\times$ " means it cannot. For the column " $\sin \theta_{13}$ ", we show the correlations between $\sin \theta_{13}$ and other observables to the leading order of $\alpha \equiv \Delta m_{21}^{2} /\left|\Delta m_{31}^{2}\right|$ and $\sin \theta_{13}$. For the column $\left|m_{e e}\right|$, we show a typical magnitudes for the averaged neutrino masses responsible for neutrino-less double beta decay. 
have 7 parameters -3 real parameters for $m_{D}$ and 4 real parameters for $M_{R}$ (one of which is a phase). As we discussed below (3.11), we can redefine the lepton fields in such a way that only one parameter in $M_{R}$ has complex phase. Following the discussion below (3.11), here also we put the phase to the matrix element $C$.

We note that this case has already been discussed as a part of the general discussion for 2 zero $m_{D}$ and 1 zero $M_{R}$ (cf. eq. (3.11) ) and the conclusions obtained i.e. $\theta_{13}=0$, inverted hierarchy and $\theta_{23}=\pi / 4$ for $b=c$ are all applicable here. After this general discussion we proceeded with detailed calculation for the $C=0$ case and found that the 4 zero texture cannot give the correct solar mixing. In this section we study the effect of $C \neq 0$. In order to study the effect of the $d^{2} C$ term, let us change the coordinate by orthogonal transformation $\widetilde{U}$;

$$
\widetilde{U}=\left(\begin{array}{ccc}
1 & 0 & 0 \\
0 & \frac{b}{\sqrt{b^{2}+c^{2}}} & \frac{-c}{\sqrt{b^{2}+c^{2}}} \\
0 & \frac{c}{\sqrt{b^{2}+c^{2}}} & \frac{b}{\sqrt{b^{2}+c^{2}}}
\end{array}\right) .
$$

Then we find

$$
\begin{aligned}
\widetilde{\mathcal{M}} & =\widetilde{U}^{\mathrm{T}} \mathcal{M} \widetilde{U} \\
& =\left(\begin{array}{ccc}
d^{2} C & \sqrt{b^{2}+c^{2}} d B & 0 \\
\sqrt{b^{2}+c^{2}} d B & \left(b^{2}+c^{2}\right) A & 0 \\
0 & 0 & 0
\end{array}\right) .
\end{aligned}
$$

Note that we are choosing the basis in which only the matrix element $C$ has a complex phase. Thus we can regard only $\mathcal{M}_{11}$ as complex valued without loss of generality. In this basis, the effect of $C \neq 0$ is clear. If the element $C$ were vanishing, there would be correlation between the mass eigenvalues and the 1-2 mixing angle in the upper-left $2 \times 2$ matrix of (3.29). However in this case the existence of $d^{2} C$ term relaxes the constraint and we can fit any mass eigenvalues and the 1-2 mixing by tuning the parameters in (3.29).

While there is one complex phase in (3.27) and (3.29), we have no chance to observe $\mathrm{CP}$ violation in the oscillation experiments as $\theta_{13}=0$. However leptogenesis [24] is possible by this phase. In the limit $\theta_{13}=0$ the effective mass constrained by $0 \nu 2 \beta$ decay $\left(\left|d^{2} C\right|\right)$ can be expressed as

$$
\left|m_{e e}\right| \simeq \sqrt{\left|\Delta m_{31}^{2}\right|} \sqrt{1-\sin ^{2} 2 \theta_{12} \sin ^{2}(\rho / 2)}
$$

which gives $\approx 0.02-0.05 \mathrm{eV}$, where the two limits correspond to $\rho=\pi$ and $\rho=0$ respectively. Thus $0 \nu 2 \beta$ will be observed in future if this texture is realized in nature [25].

Another interesting feature of (3.27) is that it provides tri-bimaximal mixing [26] under the condition where $b=c$ and $d^{2} C=2 b^{2} A-b d B$ hold. Although the latter condition implies a nontrivial correlation between Dirac and Majorana mass matrix, to build models which realize these relations might be an interesting research direction.

We note that this texture, which can be consistent with low energy phenomenology, is obtained by the choice of a non-diagonal $M_{R}$. In the $M_{R}$ diagonal basis the following relationships will hold between the elements of $m_{D}:-m_{D 11} / m_{D 12}=m_{D 22} / m_{D 21}=$ $m_{D 32} / m_{D 31}=\tan \theta_{R}$, where, $\theta_{R}$ is the angle that parametrizes the matrix $U_{R}$ in (2.4) that 
diagonalizes $M_{R}$ and can in general be expressed as

$$
U_{R}=\left(\begin{array}{cc}
\cos \theta_{R} & \sin \theta_{R} \\
-\sin \theta_{R} & \cos \theta_{R}
\end{array}\right)
$$

Hence if one considers texture zeros in $m_{D}$ with a diagonal $M_{R}$ this texture will not get included.

\subsubsection{B1. Two zero $m_{D}$ and 1 zero $M_{R}$}

Here we discuss the solution B1, where the Dirac mass matrix $m_{D}$ has 2 zero while the Majorana mass matrix $M_{R}$ has 1 zero entry;

$$
m_{D}=\left(\begin{array}{ll}
a & d \\
b & 0 \\
c & 0
\end{array}\right), \quad M_{R}^{-1}=\left(\begin{array}{cc}
A & 0 \\
0 & C
\end{array}\right) .
$$

After the seesaw integration, we find

$$
\mathcal{M}=\left(\begin{array}{lll}
a^{2} & a b & a c \\
a b & b^{2} & b c \\
a c & b c & c^{2}
\end{array}\right) A+\left(\begin{array}{ccc}
d^{2} & 0 & 0 \\
0 & 0 & 0 \\
0 & 0 & 0
\end{array}\right) C
$$

Note that we can take a basis in which only the parameter $a$ or $d$ have complex phase with no loss of generality. In spite of the existence of this un-removable phase, there is no CP violation at the low energy. One can understand this by noting that this matrix has an eigenvector $\left(0,-c / \sqrt{b^{2}+c^{2}}, b / \sqrt{b^{2}+c^{2}}\right)^{\mathrm{T}}$. Thus the same discussion holds as in the case of the solution A1 and the low energy predictions are similar.

However, the texture A1 and B1 are indeed independent. They are not associated with each other by the unitary transformation of the fields. The physical difference can arise at some high energy scale where the right-handed neutrinos are active. For example, leptogenesis or renormalization group effect-induced lepton flavor violation in supersymmetry can have different implications in the two scenarios.

It is worth mentioning that the tri-bimaximal mixing is realized if $c=b$ and $a^{2} A+d^{2} C=$ $2 b^{2} A-a b A$ hold. By trying to build models which realize this relation, we might gain insights into underlying symmetry or dynamical mechanism for the generation structure.

\subsubsection{B2. Two zero $m_{D}$ and one zero $M_{R}$}

We now consider the case where the 2 zeros in $m_{D}$ are in different columns and the one zero in the Majorana Mass matrix is in the off-diagonal position i.e. the Majorana mass matrix $M_{R}$ is diagonal;

$$
m_{D}=\left(\begin{array}{cc}
a & 0 \\
b & e \\
0 & f
\end{array}\right), \quad M_{C}^{-1}=\left(\begin{array}{cc}
A & 0 \\
0 & C
\end{array}\right) .
$$

This texture has been extensively discussed in literatures from various point of view [18]. Since the Majorana mass matrix is diagonal, only the Dirac mass matrix $m_{D}$ is responsible 
for the generation mixing. After the seesaw integration, we find

$$
\mathcal{M}=\left(\begin{array}{ccc}
a^{\prime 2} & a^{\prime} b^{\prime} & 0 \\
a^{\prime} b^{\prime} & b^{2}+c^{2} & c^{\prime} d^{\prime} \\
0 & c^{\prime} d^{\prime} & d^{\prime 2}
\end{array}\right)
$$

where we re-defined the parameters as $a^{\prime} \equiv a \sqrt{A}, b^{\prime} \equiv b \sqrt{A}, c^{\prime} \equiv e \sqrt{C}$ and $d^{\prime} \equiv f \sqrt{C}$. For the above case there are in general 12 parameters (6 angles and 6 phases). 5 phases can be absorbed in the neutrino fields. Therefore one eventually has 12 parameters $(6$ real parameters and 1 phase). Note that we can take a basis in which only the parameter $b$ or $e$ have complex phase with no loss of generality.

One of the most striking feature of this texture is that it can accommodate the normal and the inverted hierarchy simultaneously. In Table 2, this is the only solution which has such a strong flexibility. In the following we shall discuss each case in detail individually.

Normal hierarchy In the Majorana mass matrix (3.35), the large mixture for the atmospheric data and the normal mass ordering can be naturally accommodated by taking $c^{\prime} \simeq d^{\prime}$ and $c^{\prime} d^{\prime} \gg a^{\prime} b^{\prime}$. The solar angle can be nicely fitted by tuning $a^{\prime}$ and $b^{\prime}$. One of the most interesting features for this texture is that there is a connection between CP violation phenomena at high energy and low energy. Also interesting is that the prediction for $\theta_{13}$ which originates in the vanishing elements in 1-3 position;

$$
\begin{aligned}
\sin \theta_{13} & \simeq \frac{1}{2} \sin 2 \theta_{12} \tan \theta_{23} \sqrt{\alpha} \\
& =0.050-0.14
\end{aligned}
$$

where $\alpha \equiv \Delta m_{21}^{2} / \Delta m_{31}^{2}$. In the last line, we substitute $3 \sigma$ boundary values into each observables. The predicted range is very encouraging for the next generation oscillation experiments with artificial sources [27].

The effective mass $\left|m_{e e}\right|$ governing neutrino-less double beta decay for normal hierarchy can be expressed as

$$
\left|m_{e e}\right|=\left|e^{i(2 \delta+\sigma-\rho)} \sqrt{\Delta m_{21}^{2}} c_{13}^{2} s_{12}^{2}+\sqrt{\Delta m_{31}^{2}} s_{13}^{2}\right| .
$$

From the relation (3.36), we can see that the contribution from the first term is dominant in (3.37). By substituting best fit values of the solar angle and the mass differences, we obtain $\left|m_{e e}\right| \sim 0.003 \mathrm{eV}$ for the normal hierarchy.

Inverted hierarchy On the other hand, (3.35) can also accommodate the inverted mass ordering by taking

$$
\begin{aligned}
& a^{\prime 2} \simeq \sqrt{\left|\Delta m_{31}^{2}\right|} \\
& a^{\prime} b^{\prime} \simeq \sqrt{\left|\Delta m_{31}^{2}\right|-\Delta m_{21}^{2}}-\sqrt{\left|\Delta m_{31}^{2}\right|} \\
& b^{\prime 2}+c^{\prime 2}=c^{\prime} d^{\prime}=d^{\prime 2} \simeq \frac{\sqrt{\left|\Delta m_{31}^{2}\right|}}{2}
\end{aligned}
$$


After diagonalizing the 2-3 block of (3.35) by maximal mixing, we find the small 1-3 (and 3 -1) element appears as $\sim a^{\prime} b^{\prime} / \sqrt{2}$. Thus we can naively estimate the prediction for the reactor angle to be

$$
\sin \theta_{13} \simeq \frac{1}{2 \sqrt{2}} \frac{\Delta m_{21}^{2}}{\left|\Delta m_{31}^{2}\right|} \sim 0.01
$$

where we use the best fit values for the mass differences in the last line. Unfortunately the predicted magnitude is small compared to the possible reach of the next generation experiments.

It is interesting to notice that the sum of the matrix elements in each row in (3.35) is nearly the same. That is, $a^{\prime 2}+a^{\prime} b^{\prime} \simeq a^{\prime} b^{\prime}+b^{\prime 2}+c^{\prime 2}+c^{\prime} d^{\prime} \simeq c^{\prime} d^{\prime}+d^{\prime 2} \simeq \sqrt{\left|\Delta m_{31}^{2}\right|}$. This implies that we can have a tri-maximal eigenvector $(1 / \sqrt{3}, 1 / \sqrt{3}, 1 / \sqrt{3})^{\mathrm{T}}$ by tuning the parameters. Thus, the mixing matrix must be made out of the product of the tri-bimaximal mixing and a perturbation matrix;

$$
\begin{aligned}
V & =V_{\mathrm{tri}} O_{\epsilon} \\
& =\left(\begin{array}{ccc}
\frac{-2}{\sqrt{6}} & \frac{1}{\sqrt{3}} & 0 \\
\frac{1}{\sqrt{6}} & \frac{1}{\sqrt{3}} & \frac{-1}{\sqrt{2}} \\
\frac{1}{\sqrt{6}} & \frac{1}{\sqrt{3}} & \frac{1}{\sqrt{2}}
\end{array}\right)\left(\begin{array}{ccc}
\cos \theta_{\epsilon} & 0 & \sin \theta_{\epsilon} \\
0 & 1 & 0 \\
-\sin \theta_{\epsilon} & 0 & \sin \theta_{\epsilon}
\end{array}\right),
\end{aligned}
$$

where the angle $\theta_{\epsilon}$ is small as we will see in what follow. From this expression, we can infer that some generation symmetry exists behind the mass matrix (3.35). For example, it is known that the $S_{3}$ flavor symmetry and its breakdown can naturally produce the mixing form of (3.42) 28. If such a symmetric structure can account for the texture (3.35), the parameter set which is needed to produce the inverted hierarchy is no longer a group of tuned-parameters but rather unavoidable consequence of flavor symmetry and its breaking phenomena. Furthermore, we can use the expression (3.42) for more practical purposes. For example, we can obtain more precise expression for $\theta_{13}$ than the rough estimation (3.41) by fixing the angle $\theta_{\epsilon}$ in (3.42). By reconstructing the mass matrix in terms of the $V$ in (3.42) and the diagonal mass eigenvalues $\operatorname{diag}\left(\sqrt{\left|\Delta m_{31}^{2}\right|}, \sqrt{\left|\Delta m_{31}^{2}\right|+\Delta m_{21}^{2}}, 0\right)$, and imposing the texture zero condition for the 1-3 entry, we find

$$
\frac{m_{2}}{3}-\sqrt{\frac{2}{3}} \cos \theta_{\epsilon}\left(\frac{\cos \theta_{\epsilon}}{\sqrt{6}}-\frac{\sin \theta_{\epsilon}}{\sqrt{2}}\right) m_{1}=0
$$

where $m_{1}=\sqrt{\left|\Delta m_{31}^{2}\right|}$ and $m_{2}=\sqrt{\left|\Delta m_{31}^{2}\right|+\Delta m_{21}^{2}}$. From this equation we can fix the perturbation $\theta_{\epsilon}$ as

$$
\sin \theta_{\epsilon} \simeq \frac{\alpha}{2 \sqrt{3}}
$$

where $\alpha \equiv \Delta m_{21}^{2} /\left|\Delta m_{31}^{2}\right|$. Here we choose the smaller solution of the equation (3.43) for fitting the $\theta_{13}$. From (3.44) we find that $\sin \theta_{13}=\frac{\alpha}{3 \sqrt{2}}$ which agrees well with (3.41).

The above prediction is interesting in the sense that it is associated with the tribimaximal mixing and its deviation. However we can expand (3.44) to more general formula. As we have done in the case of the normal hierarchy, we can write the Majorana mass matrix in terms of the mixing angles and the mass eigenvalues, and derive the 
condition for the 1-3 and 3-1 vanishing elements. Then we find

$$
-m_{1} c_{12}^{2} s_{13} \cos \delta+m_{1} c_{12} s_{12} t_{23}-m_{2} s_{12}^{2} s_{13} \cos (\delta+\rho)-m_{2} c_{12} s_{12} t_{23} \cos \rho=0,
$$

and

$$
-m_{1} c_{12}^{2} s_{13} \sin \delta-m_{2} s_{12}^{2} s_{13} \sin (\delta+\rho)-m_{2} c_{12} s_{12} t_{23} \sin \rho=0 .
$$

where $t_{23} \equiv \tan \theta_{23}, \delta$ is the Dirac phase and $\rho$ stands for the Majorana phase. From (3.46), we can see that $\delta=\rho=\mathcal{O}\left(s_{13}\right)$ must hold in order to keep the texture zero in 1-3 position. Then (3.45) gives

$$
\sin \theta_{13}=\frac{\alpha}{4} \sin 2 \theta_{12} \tan \theta_{23}
$$

as a leading order relation in $\alpha$ and $\sin \theta_{13}$. Note that at the tri-bimaximal limit $\sin 2 \theta_{12} \rightarrow$ $\frac{2 \sqrt{2}}{3}$ and $\tan \theta_{23} \rightarrow 1$, we can reproduce the previous formula of $\sin \theta_{13}=\frac{\alpha}{3 \sqrt{2}}$. For inverted hierarchy, the effective mass $m_{e e}$ measured in neutrino-less double beta decay can be expressed as in (3.30), which gives relatively large mass parameter $\left|m_{e e}\right| \sim 0.05 \mathrm{eV}$ because of the small Majorana phase of $\rho=\mathcal{O}\left(s_{13}\right)$.

The $\mathrm{CP}$ violating phases and their connection to baryon number asymmetry of the universe for this texture was considered in literature in [18].

\subsubsection{B3. Two zero $m_{D}$ and one zero $M_{R}$}

Here we discuss the solution B3, where the Dirac mass matrix $m_{D}$ has 2 zero while the Majorana mass matrix $M_{C}$ has 1 zero entry;

$$
m_{D}=\left(\begin{array}{ll}
a & d \\
b & 0 \\
c & 0
\end{array}\right), \quad M_{C}^{-1}=\left(\begin{array}{cc}
A & B \\
B & 0
\end{array}\right) .
$$

After the seesaw operation, we find

$$
\mathcal{M}=\left(\begin{array}{lll}
a^{2} & a b & a c \\
a b & b^{2} & b c \\
a c & b c & c^{2}
\end{array}\right) A+\left(\begin{array}{ccc}
2 a d & b d & c d \\
b d & 0 & 0 \\
c d & 0 & 0
\end{array}\right) B
$$

Note that we can take a basis in which only the parameter $a$ or $d$ have complex phase with no loss of generality. It is useful to notice that this matrix has an eigenvector $\left(0,-c / \sqrt{b^{2}+c^{2}}, b / \sqrt{b^{2}+c^{2}}\right)^{\mathrm{T}}$. Thus the same discussion, as in the case of the solution $\mathrm{A} 1$ and $\mathrm{B} 1$, remain valid leading to similar low energy predictions.

As the solutions $\mathrm{A} 1$ and $\mathrm{B} 1$, this texture can also provide the tri-bimaximal mixing. It is realized if $c=b$ and $a^{2} A+2 a d B=2 b^{2} A-(a b A+b d B)$ hold.

Note that in this case the $M_{R}$ diagonal basis implies the following relation between the elements $m_{D 22} / m_{D 21}=m_{D 32} / m_{D 31}=\tan \theta_{R}$ where $\theta_{R}$ is the angle parametrizing $U_{R}$ as in (3.31). Thus in the $M_{R}$ diagonal basis the zeros of $m_{D}$ will not be visible. 


\subsubsection{B4. Two zero $m_{D}$ and one zero $M_{R}$}

Here we discuss the texture B4, where the Majorana mass matrix has texture zero in 1-1 position;

$$
m_{D}=\left(\begin{array}{cc}
a & 0 \\
0 & e \\
c & f
\end{array}\right), \quad M_{C}^{-1}=\left(\begin{array}{cc}
0 & B \\
B & C
\end{array}\right) .
$$

For this case there are $8+4=12$ real parameters characterizing $m_{D}$ and $M_{R}$. The 2 phases in $M_{R}$ can be absorbed by redefining the fields and one can consider $M_{R}$ to be real. 3 phases in $m_{D}$ can likewise be removed leaving 5 parameters one of which is a phase. Note that we can take a basis in which only the parameter $c$ or $f$ have complex phase with no loss of generality. After the seesaw integration, we find the induced Majorana mass matrix to be

$$
\mathcal{M}=\left(\begin{array}{ccc}
0 & a^{\prime} e^{\prime} & a^{\prime} f^{\prime} \\
a^{\prime} e^{\prime} & 0 & c^{\prime} e^{\prime} \\
a^{\prime} f^{\prime} & c^{\prime} e^{\prime} & 2 c^{\prime} f^{\prime}
\end{array}\right)+\left(\begin{array}{ccc}
0 & 0 & 0 \\
0 & e^{\prime 2} & e^{\prime} f^{\prime} \\
0 & e^{\prime} f^{\prime} & f^{\prime 2}
\end{array}\right)
$$

where the right-handed parameters are absorbed as $e^{\prime} \equiv e \sqrt{C}, f^{\prime} \equiv f \sqrt{C}, a^{\prime} \equiv a B / \sqrt{C}$, $c^{\prime} \equiv c B / \sqrt{C}$.

From (3.51), we can see that 4 effective parameters $a^{\prime}, c^{\prime}, e^{\prime}, f^{\prime}$ control the low-energy physics. Thus there should be one relation among the 5 observables. As a consequence of the fact that the 1-1 element is vanishing and $m_{1}=0$, we have the prediction

$$
\sin \theta_{13} \simeq \alpha^{\frac{1}{4}} \sin \theta_{12}
$$

which is the same as the prediction of the 4 zero textures (3.18). For the texture (3.18), we have found that $\sin \theta_{23}$ is predicted to be too small compared to the observed large mixture. However, in the texture (3.51), we have a correction matrix in 2-3 sector against the first term which is the same form as (3.18). We already have one relation of (3.52) so that $\sin \theta_{23}$ can be fitted by tuning the original mass matrix parameters. The $\left|m_{e e}\right|$ is $\sim$ 0 in this case which is beyond the reach of the next generation neutrino-less double beta experiments.

As we have discussed in the texture (3.18), the correlation (3.52) requires marginal values of the mass differences and the solar angle in order that the reactor angle is inside the present $3 \sigma$ allowed range. Together with the improvement of the data about $\theta_{13}$, precise measurements for the mass differences and the solar angle can judge this texture in near future.

In this case the $M_{R}$ diagonal basis would imply the following relation between the elements $-m_{D 21} / m_{D 22}=m_{D 12} / m_{D 11}=\tan \theta_{R}$ where $\theta_{R}$ is the angle parametrizing $U_{R}$ as in (3.31). 


\subsubsection{C1. One zero $m_{D}$ and two zero $M_{R}$}

Here we discuss the texture $\mathrm{C} 1$, where the Dirac mass matrix has 1 zero and the Majorana mass matrix has 2 zeros;

$$
m_{D}=\left(\begin{array}{cc}
a & 0 \\
b & e \\
c & f
\end{array}\right), \quad M_{C}^{-1}=\left(\begin{array}{cc}
0 & B \\
B & 0
\end{array}\right) .
$$

In this case there are 6 parameters ( 1 of which is phase) characterizing $m_{D}$ and 1 parameter for $M_{R}$. We can take a basis in which only $b, e, c$ or $f$ has complex phase with no loss of generality. After the seesaw integration, we find

$$
\mathcal{M}=\left(\begin{array}{ccc}
0 & a^{\prime} e^{\prime} & a^{\prime} f^{\prime} \\
a^{\prime} e^{\prime} & 0 & c^{\prime} e^{\prime} \\
a^{\prime} f^{\prime} & c^{\prime} e^{\prime} & 2 c^{\prime} f^{\prime}
\end{array}\right)+\left(\begin{array}{ccc}
0 & 0 & 0 \\
0 & 2 e^{\prime} b^{\prime} & b^{\prime} f^{\prime} \\
0 & b^{\prime} f^{\prime} & 0
\end{array}\right)
$$

where the right-handed parameters are absorbed as $a^{\prime} \equiv a \sqrt{B}, b^{\prime} \equiv b \sqrt{B}, c^{\prime}=c \sqrt{B}$, $e^{\prime} \equiv e \sqrt{B}$

It is immediately seen that the texture zero in the 1-1 position leads to the same prediction as that of B4 for the normal hierarchy (3.52). Moreover, as in the texture B4, we have a correction matrix in 2-3 sector against the Majorana mass matrix which is the resultant form of (3.18) after seesaw operation, though the structure of the correction is different from that of B4. It turns out however that the second term (3.54) can also help to fit $\sin \theta_{23}$, so that there is no difference between the low energy predictions of B4 and C1.

In this case the $M_{R}$ diagonal basis would imply the following relation between the elements of $m_{D}: m_{D 12} / m_{D 11}=\tan \theta_{R}$ where $\theta_{R}$ is the angle parametrizing $U_{R}$ as in (3.31).

\subsubsection{C2: One zero $m_{D}$ and two zero $M_{R}$}

Here we discuss the texture $\mathrm{C} 2$, which is the last option in Table 2 .

$$
m_{D}=\left(\begin{array}{ll}
a & d \\
b & 0 \\
c & f
\end{array}\right), \quad M_{C}^{-1}=\left(\begin{array}{cc}
0 & B \\
B & 0
\end{array}\right) .
$$

This texture is obtained by exchanging 1-2 rows of $m_{D}$ in (3.53), so that we can obtain the low-energy Majorana mass matrix by permuting the 1-2 rows and the columns of (3.54) with replacements $b \rightarrow a, a \rightarrow b$ and $e \rightarrow d$. As in the solution $\mathrm{C} 1$, we can take the basis in which the matrix element $a, d, c$ or $f$ has complex phase.

It turns out that this texture is viable only with the inverted hierarchy. Since the effective Majorana mass matrix is obtained by 1-2 exchange of the rows and the columns of (3.54), there is one texture zero in $2-2$ position. We thus have one correlation among masses and mixings. We find the sum rule

$$
\sin \theta_{12} \simeq \frac{1}{\sqrt{2}}-\frac{1}{8 \sqrt{2}} \alpha-\frac{1}{\sqrt{2}} \tan \theta_{23} \sin \theta_{13}
$$


holds. Here $\alpha \equiv \Delta m_{21}^{2} /\left|\Delta m_{31}^{2}\right|$ and this relation is given as the leading order approximation in powers of $\alpha$ and $\sin \theta_{13}$. The phase parameters are to be fixed as $\rho=\pi$ and $\delta=0$ up to this order. Note that we can see this equation as a modified formula for (3.14) which leads to too large solar mixing. Now the problem is ameliorated by the existence of the third term. If $\theta_{13}=0$ then this texture cannot give the correct solar angle.

By setting $\alpha \rightarrow 0$ and $\tan \theta_{23} \rightarrow 1$, we find (3.56) implies

$$
\sin \theta_{13} \simeq 1-\sqrt{2} \sin \theta_{12}=0.20_{-0.1}^{+0.08} .
$$

In the right hand side we used the best fit and $3 \sigma$ boundary values for the $\sin \theta_{12}$. The reactor angle must be just below the present $3 \sigma$ upper bound. The future reactor experiment will confirm $\sin \theta_{13}$ of $\mathcal{O}\left(10^{-1}\right)$ if this texture is realized.

Moreover, it is interesting to note that 1-1 element of $\mathcal{M}$ is relatively large because of the inverted mass spectrum:

$$
\left|m_{e e}\right| \simeq \sqrt{\left|\Delta m_{31}^{2}\right|} \cos 2 \theta_{12} \sim 0.02 \mathrm{eV} .
$$

Thus, with this texture form, we have a good chance to confirm that the neutrinos are indeed Majorana particles in near future [25]. The most prominent feature is that in both measurements - the reactor angle and neutrino-less double beta decay - we will find positive signals simultaneously at the next generation facilities.

Finally we would like to comment on the $\mathrm{CP}$ violation. As we mentioned above, there is one phase which cannot be removed by the redefinition of the fields. This one phase controls all $\mathrm{CP}$ violation phenomena at high energy as well as low energy. It is interesting to observe that the heavy neutrino masses are degenerate in (3.55). Although the lepton asymmetry vanish with exact degeneracy, the degeneracy may be relaxed, for example, by radiative corrections from the other sector. This fact may lead to enhanced lepton asymmetry by the contribution from the self-energy diagram [29]. The baryon number of the universe will be proportional to $\operatorname{Im}\left(m_{D}^{\dagger} m_{D}\right)_{12}^{2}=-2 a d \mathcal{X} \sin \phi$, where $\phi$ is the phase (of $a$ or $d$ ) and $\mathcal{X}=|a|^{2}+|b|^{2}+|c|^{2}-|d|^{2}-|f|^{2}$. On the other hand the weak basis invariant 30. responsible for low energy $\mathrm{CP}$ violation is found to be proportional to $(\mathcal{F} \cos \phi+\mathcal{G}) \sin \phi$ where $\mathcal{F}$ and $\mathcal{G}$ are some functions of the elements of $m_{D}$. The detailed analysis of $\mathrm{CP}$ violation and the models which produce the texture (3.55) will be presented in a separate paper [31].

In this case the $M_{R}$ diagonal basis would imply the following relation between the elements of $m_{D}: m_{D 22} / m_{D 21}=\tan \theta_{R}$ where $\theta_{R}$ is the angle parametrizing $U_{R}$ as in (3.31).

\section{Summary and Conclusions}

In this paper we analyze the texture zeros in the neutrino Yukawa Coupling matrix $m_{D}$ and the heavy Majorana neutrino mass matrix $M_{R}$ in the context of the minimal seesaw model including 2 heavy right-handed neutrinos. We illustrate which textures are compatible with the present neutrino oscillation data and discuss their implications for the future neutrino experiments. We do not make the assumption that $M_{R}$ is diagonal.

We first consider the zeros in the neutrino Dirac mass matrix $m_{D}$ and show that it cannot have 4 or more zeros. Thus the maximum number of zeros $m_{D}$ can have is 3 . For 
the Majorana mass matrix of the right-handed neutrinos $M_{R}$, the maximum number of zeros can be 2. Except for the case where $M_{R}$ has vanishing determinant, the possible texture in this case is zeros in the diagonal position. Thus the maximal number of zeros that can be allowed in $m_{D}$ and $M_{R}$ taken together are 5 (3 zeros in $m_{D}$ and 2 zeros in $M_{R}$ ). But such a pattern give rise to a Majorana mass matrix with more than two zeros which is incompatible with data according to [11.

If we consider total 4 zeros in $m_{D}$ and $M_{R}$ then there are two possibilities :

(i) 3 zeros in $m_{D}$ and 1 zero in $M_{R}$

(ii) 2 zeros in $m_{D}$ and 2 zeros in $M_{R}$

The pattern (i) gives rise to the inverted mass ordering with $m_{3}=0, \theta_{13}=0$ but the other angles are close to bimaximal mixing. The pattern (ii) can accommodate both normal and inverted hierarchy but it fails to predict one mixing angle. For the normal hierarchy, the atmospheric angle $\theta_{23}$ is too large or too small. For the inverted hierarchy, it gives bi-maximal mixing which is not consistent with the current data which dictates $\theta_{12}$ to be close to $33^{\circ}$. Thus at this level also there are no acceptable solutions.

At the next tier we consider total 3 zeros in $m_{D}$ and $M_{R}$. The possibilities in these cases are

(i) 3 zeros in $m_{D}$ and no zero in $M_{R}$

(ii) 2 zeros in $m_{D}$ and 1 zero in $M_{R}$

(iii) 1 zero in $m_{D}$ and 2 zeros in $M_{R}$

By exhausting all texture possibilities in each category, we found seven patterns which are viable with the current data. This is summarized in Table 2. All these patterns can accommodate either normal hierarchy or inverted hierarchy or both and gives definite predictions for $\theta_{13}$ which can be testable in the near future.

For case (i) with 3 zeros in $m_{D}$ and no zeros in $M_{R}$ it is possible to get inverted hierarchy with $\theta_{13}=0$. It is also possible to get tri-bimaximal mixing under certain conditions on the elements of $m_{D}$ and $M_{R}$.

For case (ii) there are two classes of patterns - one in which both zeros in $m_{D}$ appear in the same column and the other in which the two zeros are placed in different columns. In both cases assuming a diagonal $M_{R}$ it is possible to reproduce the current low energy data. The pattern with zeros in same column of $m_{D}$ can accommodate only inverted hierarchy and predicts $\theta_{13}=0$. Again tri-bimaximal mixing is reproduced if certain equalities involving the elements of $m_{D}$ and $M_{R}$ are obeyed. The case where the zeros appear in different columns can accommodate both normal and inverted hierarchy. There are definite predictions for $\theta_{13}$ for both normal and inverted case which can be tested in near future experiments. In this category, we also include the possibility where one of the diagonal entries of $M_{R}$ is zero. In this situation, for the case where the two zeros of $m_{D}$ appear in the same column, one gets inverted hierarchy with $\sin \theta_{13}=0$. Whereas for $m_{D}$ with the 2 zeros in different columns one gets normal hierarchy with a definite non-zero prediction for $\theta_{13}$ in terms of $\Delta m_{21}^{2} / \Delta m_{31}^{2}$ and $\theta_{12}$.

For case (iii) of 1 zero $m_{D}$ and 2 zero $M_{R}$ we find two allowed patterns viable with data. Both these cases are for non-diagonal $M_{R}$ with definite non-zero predictions for $\theta_{13}$.

Summarizing, out of the 7 allowed patterns, 5 arise because we have relaxed the assumption of a diagonal $M_{R}$. In a basis where $M_{R}$ is diagonal the zeros in $m_{D}$ for all these 5 patterns get hidden as specific relations between the different elements of $m_{D}$. To the best of our knowledge, these textures which are viable with the current data, have escaped 
attention since most analysis of texture zeros in literature had been done in a basis in which $M_{R}$ is diagonal, or avoided mentioning the mass textures which do not have definite correlations among observable parameters.

The mass matrix list of Table 2 presents the most economical extensions of the standard model. The 7 solutions are minimal solutions for neutrino physics known at present in terms of not only the number of the parameters but also the field content. It is rather surprising that such simple economy solely leads to the rich predictions shown in Table 2 Although the mass textures themselves can be discriminated only by precise measurements of the low energy parameters, the mechanism which realizes particular texture might provide further predictions and/or new phenomena which can be targets of next generation neutrino physics, collider physics, astrophysics and so on. We hope that the 7 possibilities and preceded almost viable forms provide a foundation of model building which yields testable predictions and deeper understanding of the generation structure.

\section{Acknowledgments}

The authors acknowledge support from the neutrino project under the XI plan of the Harish-Chandra Research Institute. S.G. wishes to thank Anjan Joshipura for insightful discussions. A.W. is grateful to the organizers and participants of the conference "NuHoRIzons" held at Harish-Chandra Research Institute, Allahabad, India on 13-15 February 2008, for discussions leading to this work. The authors thank Probir Roy for his involvement in the initial phase of the work.

\section{References}

[1] For a recent review see M. C. Gonzalez-Garcia and M. Maltoni, Phys. Rept. 460, 1 (2008) arXiv:0704.1800 [hep-ph]].

[2] M. Maltoni, T. Schwetz, M. A. Tortola and J. W. F. Valle, New J. Phys. 6, 122 (2004) arXiv:hep-ph/0405172, version 6.

[3] C. Kraus et al., Eur. Phys. J. C 40, 447 (2005). arXiv:hep-ex/0412056.

[4] J. Schechter and J. W. F. Valle, Phys. Rev. D 22, 2227 (1980).

[5] H. V. Klapdor-Kleingrothaus et al., Eur. Phys. J. A 12, 147 (2001).

[6] C. E. Aalseth et al. [IGEX Collaboration], Phys. Rev. D 65, 092007 (2002).

[7] P. Minkowski, Phys. Lett. B 67, 421 (1977); T. Yanagida, in Proceedings of the Workshop on the Unified Theory and the Baryon Number in the Universe (O. Sawada and A. Sugamoto, eds.), KEK, Tsukuba, Japan, 1979, p. 95; M. Gell-Mann, P. Ramond, and R. Slansky, Complex spinors and unified theories, in Supergravity (P. van Nieuwenhuizen and D. Z. Freedman, eds.), North Holland, Amsterdam, 1979, p. 315; S. L. Glashow, The future of elementary particle physics, in Proceedings of the 1979 Cargèse Summer Institute on Quarks and Leptons (M. Lévy, J.-L. Basdevant, D. Speiser, 
J. Weyers, R. Gastmans, and M. Jacob, eds.), Plenum Press, New York, 1980, pp. 687713.

[8] R. N. Mohapatra and G. Senjanovic, Phys. Rev. Lett. 44, 912 (1980).

[9] M. Magg and C. Wetterich, Phys. Lett. B 94, 61 (1980); G. Lazarides, Q. Shafi and C. Wetterich, Nucl. Phys. B 181, 287 (1981).

[10] R. Foot, H. Lew, X. G. He and G. C. Joshi, Z. Phys. C 44, 441 (1989).

[11] P. H. Frampton, S. L. Glashow and D. Marfatia, Phys. Lett. B 536, 79 (2002) arXiv:hep-ph/0201008].

[12] Z. z. Xing, Phys. Lett. B 530, 159 (2002) arXiv:hep-ph/0201151; Z. z. Xing, Phys. Lett. B 539, 85 (2002) arXiv:hep-ph/0205032]; B. R. Desai, D. P. Roy and A. R. Vaucher, Mod. Phys. Lett. A 18, 1355 (2003) [arXiv:hep-ph/0209035]; A. Merle and W. Rodejohann, Phys. Rev. D 73, 073012 (2006) [arXiv:hep-ph/0603111; S. Dev, S. Kumar, S. Verma and S. Gupta, Phys. Rev. D 76, 013002 (2007) arXiv:hep-ph/0612102.

[13] H. Fritzsch, Phys. Lett. B 73, 317 (1978); G. F. Giudice, Mod. Phys. Lett. A 7, 2429 (1992) arXiv:hep-ph/9204215]; P. Ramond, R. G. Roberts and G. G. Ross, Nucl. Phys. B 406, 19 (1993) [arXiv:hep-ph/9303320]; G. C. Branco and J. I. SilvaMarcos, Phys. Lett. B 331, 390 (1994); T. K. Kuo, S. W. Mansour and G. H. Wu, Phys. Rev. D 60, 093004 (1999) arXiv:hep-ph/9907314]; H. Fritzsch and Z. z. Xing, Prog. Part. Nucl. Phys. 45, 1 (2000) arXiv:hep-ph/9912358]; R. G. Roberts, A. Romanino, G. G. Ross and L. Velasco-Sevilla, Nucl. Phys. B 615, 358 (2001) arXiv:hep-ph/0104088]; H. D. Kim, S. Raby and L. Schradin, Phys. Rev. D 69, 092002 (2004) |arXiv:hep-ph/0401169]; N. Uekusa, A. Watanabe and K. Yoshioka, Phys. Rev. D 71, 094024 (2005) [arXiv:hep-ph/0501211]; S. Tatur and J. Bartelski, Phys. Rev. D 74, 013007 (2006) arXiv:hep-ph/0605261].

[14] C. D. Froggatt and H. B. Nielsen, Nucl. Phys. B 147, 277 (1979).

[15] W. Grimus, A. S. Joshipura, L. Lavoura and M. Tanimoto, Eur. Phys. J. C 36, 227 (2004) arXiv:hep-ph/0405016]; N. Haba and K. Yoshioka, Nucl. Phys. B 739, 254 (2006) arXiv:hep-ph/0511108; S. Kaneko, H. Sawanaka, T. Shingai, M. Tanimoto and K. Yoshioka, Prog. Theor. Phys. 117, 161 (2007) arXiv:hep-ph/0609220]; S. Kaneko, H. Sawanaka, T. Shingai, M. Tanimoto and K. Yoshioka, [arXiv:hep-ph/0703250].

[16] G. Bhattacharyya, A. Raychaudhuri and A. Sil, Phys. Rev. D 67, 073004 (2003) arXiv:hep-ph/0211074]; C. Hagedorn, J. Kersten and M. Lindner, Phys. Lett. B 597, 63 (2004) arXiv:hep-ph/0406103]; M. Honda, S. Kaneko and M. Tanimoto, JHEP 0309, 028 (2003) [arXiv:hep-ph/0303227.

[17] G. K. Leontaris, S. Lola, C. Scheich and J. D. Vergados, Phys. Rev. D 53, 6381 (1996) arXiv:hep-ph/9509351; S. M. Barr and I. Dorsner, Nucl. Phys. B 585, 79 (2000) arXiv:hep-ph/0003058; A. Kageyama, S. Kaneko, N. Shimoyama and M. Tanimoto, Phys. Lett. B 538, 96 (2002) arXiv:hep-ph/0204291; P. H. Frampton, S. L. Glashow and T. Yanagida, Phys. Lett. B 548, 119 (2002) 
arXiv:hep-ph/0208157]; R. Barbieri, T. Hambye and A. Romanino, JHEP 0303, 017 (2003) arXiv:hep-ph/0302118]; A. Ibarra and G. G. Ross, Phys. Lett. B 591, 285 (2004) arXiv:hep-ph/0312138; S. Chang, S. K. Kang and K. Siyeon, Phys. Lett. B 597, 78 (2004) [arXiv:hep-ph/0404187]; C. Hagedorn and W. Rodejohann, JHEP 0507, 034 (2005) arXiv:hep-ph/0503143]; A. Watanabe and K. Yoshioka, JHEP 0605, 044 (2006) arXiv:hep-ph/0601152]; W. 1. Guo, Z. z. Xing and S. Zhou, Int. J. Mod. Phys. E 16, 1 (2007) [arXiv:hep-ph/0612033]; G. C. Branco, D. Emmanuel-Costa, M. N. Rebelo and P. Roy, Phys. Rev. D 77, 053011 (2008) [arXiv:0712.0774 [hep-ph]].

[18] S. F. King, Phys. Lett. B 439, 350 (1998) arXiv:hep-ph/9806440; S. F. King, Nucl. Phys. B 562, 57 (1999) arXiv:hep-ph/9904210]; R. Kuchimanchi and R. N. Mohapatra, Phys. Rev. D 66, 051301 (2002) [arXiv:hep-ph/0207110]; T. Endoh, S. Kaneko, S. K. Kang, T. Morozumi and M. Tanimoto, Phys. Rev. Lett. 89, 231601 (2002) [arXiv:hep-ph/0209020]; M. Raidal and A. Strumia, Phys. Lett. B 553, 72 (2003) |arXiv:hep-ph/0210021]; S. F. King, Phys. Rev. D 67, 113010 (2003) arXiv:hep-ph/0211228]; S. Raby, Phys. Lett. B 561, 119 (2003) arXiv:hep-ph/0302027]; B. Dutta and R. N. Mohapatra, Phys. Rev. D 68, 056006 (2003) arXiv:hep-ph/0305059]; V. Barger, D. A. Dicus, H. J. He and T. j. Li, Phys. Lett. B 583, 173 (2004) arXiv:hep-ph/0310278]; W. l. Guo and Z. z. Xing, Phys. Lett. B 583, 163 (2004) arXiv:hep-ph/0310326]; W. Rodejohann, Eur. Phys. J. C 32, 235 (2004) arXiv:hep-ph/0311142]; K. Bhattacharya, N. Sahu, U. Sarkar and S. K. Singh, Phys. Rev. D 74, 093001 (2006) arXiv:hep-ph/0607272 ; B. Brahmachari and N. Okada, Phys. Lett. B 660, 508 (2008) [arXiv:hep-ph/0612079].

[19] R. Barbieri, T. Hambye and A. Romanino; A. Ibarra and G. G. Ross; W. l. Guo, Z. z. Xing and S. Zhou; S. Chang, S. K. Kang and K. Siyeon in Ref. [17].

[20] K. S. Babu and S. M. Barr, Phys. Lett. B 381, 202 (1996) arXiv:hep-ph/9511446; J. Sato and T. Yanagida, Phys. Lett. B 430, 127 (1998) arXiv:hep-ph/9710516; C. H. Albright, K. S. Babu and S. M. Barr, Phys. Rev. Lett. 81, 1167 (1998) arXiv:hep-ph/9802314]; J. K. Elwood, N. Irges and P. Ramond, Phys. Rev. Lett. 81, 5064 (1998) [arXiv:hep-ph/9807228]; G. Altarelli and F. Feruglio, Phys. Lett. B 451, 388 (1999) arXiv:hep-ph/9812475; Q. Shafi and Z. Tavartkiladze, Phys. Lett. B 487, 145 (2000) arXiv:hep-ph/9910314 ; P. H. Frampton and A. Rasin, Phys. Lett. B 478, 424 (2000) arXiv:hep-ph/9910522 ; M. Bando, T. Kugo and K. Yoshioka, Prog. Theor. Phys. 104, 211 (2000) arXiv:hep-ph/0003220]; X. J. Bi and Y. B. Dai, Phys. Rev. D 66, 076006 (2002) arXiv:hep-ph/0112077.

[21] W. Grimus, A. S. Joshipura, S. Kaneko, L. Lavoura, H. Sawanaka and M. Tanimoto, Nucl. Phys. B 713, 151 (2005) arXiv:hep-ph/0408123.

[22] A. Bandyopadhyay, S. Choubey, S. Goswami, S. T. Petcov and D. P. Roy, Phys. Lett. B 583, 134 (2004) arXiv:hep-ph/0309174.

[23] S. Antusch, J. Kersten, M. Lindner and M. Ratz, Phys. Lett. B 544, 1 (2002) arXiv:hep-ph/0206078.

[24] M. Fukugita and T. Yanagida, Phys. Lett. B 174, 45 (1986). 
[25] C. Aalseth et al., hep-ph/0412300.

[26] P. F. Harrison, D. H. Perkins and W. G. Scott, Phys. Lett. B 530, 167 (2002) arXiv:hep-ph/0202074]; P. F. Harrison and W. G. Scott, Phys. Lett. B 535, 163 (2002) arXiv:hep-ph/0203209.

[27] Letter of Intent for Double-CHOOZ F. Ardellier et. al., hep-ex/0405032.

[28] N. Haba, A. Watanabe and K. Yoshioka, Phys. Rev. Lett. 97, 041601 (2006) arXiv:hep-ph/0603116.

[29] L. Covi, E. Roulet and F. Vissani, Phys. Lett. B 384, 169 (1996) arXiv:hep-ph/9605319; M. Flanz, E. A. Paschos, U. Sarkar and J. Weiss, Phys. Lett. B 389, 693 (1996) arXiv:hep-ph/9607310; A. Pilaftsis, Nucl. Phys. B 504, 61 (1997) arXiv:hep-ph/9702393; A. Pilaftsis, Phys. Rev. D 56, 5431 (1997) arXiv:hep-ph/9707235]; G. F. Giudice, A. Notari, M. Raidal, A. Riotto and A. Strumia, Nucl. Phys. B 685, 89 (2004) arXiv:hep-ph/0310123.

[30] C. Jarlskog, Phys. Rev. Lett. 55, 1039 (1985); G. C. Branco, T. Morozumi, B. M. Nobre and M. N. Rebelo, Nucl. Phys. B 617, 475 (2001) arXiv:hep-ph/0107164; G. C. Branco, M. N. Rebelo and J. I. Silva-Marcos, Phys. Lett. B 633, 345 (2006) arXiv:hep-ph/0510412.

[31] S. Goswami, P. Roy, A. Watanabe in preparation. 